\title{
A systematic surgical procedure: The ' $7+3$ ' approach to laparoscopic right partial hepatectomy [deep segment (S) VI, S VII or S VIII] in 52 patients with liver tumors
}

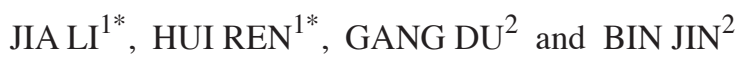 \\ ${ }^{1}$ Department of Liver Transplantation Surgery, 302 Military Hospital of China, Beijing 100039; \\ ${ }^{2}$ Department of General Surgery, Qilu Hospital of Shandong University, Jinan, Shandong 250012, P.R. China
}

Received September 13, 2017; Accepted February 15, 2018

DOI: $10.3892 / 01.2018 .8345$

\begin{abstract}
Laparoscopic right partial hepatectomy (LRPH), located in the deep segment (S) VI, S VII or S VIII, is a complicated procedure, due to its poor operative field and high risk of bleeding. The present study aimed to summarize our experience of LRPH and to share our systematic surgical procedure, the '7+3' approach. This approach includes seven key points and three main instruments. A total of 81 cases were included, which were divided into 2 groups [LRPH, $\mathrm{n}=52$; open hepatectomy $(\mathrm{OH}), \mathrm{n}=29$ ]. The demographic profile, intraoperative parameters and postoperative parameters were obtained and analyzed. Blood loss $(245.38 \pm 268.37 \mathrm{ml})$ in the LRPH group was not significantly more than in the $\mathrm{OH}$ group $(230.93 \pm 257.62 \mathrm{ml} ; \mathrm{P}=0.936)$. The durations of surgery, liver parenchyma transection and portal triad clamping were also not significantly more than those in the $\mathrm{OH}$ group (145.52 \pm 48.29 vs. $129.83 \pm 35.04 \mathrm{~min} ; \mathrm{P}=0.149$ for surgery; $28.52 \pm 10.16$ vs. $23.97 \pm 10.44 \mathrm{~min} ; \mathrm{P}=0.059$ for liver parenchyma transection; $20.62 \pm 9.61$ vs. $17.31 \pm 10.12 \mathrm{~min}$; $\mathrm{P}=0.149$ for portal triad clamping). However, the number of postoperative hospital days in the LRPH group was smaller (10.67 in LRPH vs. 12.07 in $\mathrm{OH} ; \mathrm{P}=0.025)$. The present study demonstrated the satisfactory surgical outcomes and economic benefits of the systematic '7+3' surgical technique for LRPH. Further studies in larger cohorts and other centers are required to confirm its feasibility and superiority.
\end{abstract}

Correspondence to: Professor Bin Jin, Department of General Surgery, Qilu Hospital of Shandong University, 107 Wenhua West Road, Jinan, Shandong 250012, P.R. China

E-mail: jinbin9449@126.com

*Contributed equally

Abbreviations: LRPH, laparoscopic right partial hepatectomy; LS, laparoscopic surgery; LH, laparoscopic hepatectomy; OH, open hepatectomy; ICCLLR, International Consensus Conference on Laparoscopic Liver Resection

Key words: systematic approach, laparoscopic surgery, laparoscopic right partial hepatectomy, experience

\section{Introduction}

As the limitations of laparoscopic equipment and surgical techniques are being gradually overcome, indications for laparoscopic surgery (LS) have been extended from diagnostic therapy to radical therapy, from benign disease to malignant lesions, and from cavity viscera to solid visceral extensions. Laparoscopic hepatectomy (LH), a typical LS procedure, has been rapidly and widely applied in the field of abdominal surgery, due to its advantages associated with minimally-invasive surgery (1-5). As of 2016, LH has been increasing in frequency, with a total of $>9,000 \mathrm{LH}$ procedures having been performed worldwide to date; however, the majority of these were applied to local resections in antero-lateral segments of the liver, with only 1,297 of these being right hepatectomies (13.6\%), and only 15 and 19 being right anterior lobectomies and right posterior lobectomies, respectively, according to Ciria et al (6). LH for tumors in the right side of the liver is one of the most recent challenges to be discussed in this field. These tumors are located in the bottom of the subphrenic rib cage, overlaid by the large and heavy right side of the liver when the patient is in the supine position. LH of this area is technically demanding in the handling of the large and heavy right side of the liver in the small subphrenic rib cage; and obtaining a fine surgical view is very challenging, as is the manipulation to ensure hemostasis and obtainment of appropriate surgical margins. Previous studies $(7,8)$ have reported on the significant technical challenges of laparoscopic right partial hepatectomy (LRPH), located in the deep segment (S) VI, S VII or S VIII. Numerous associated technologies, including anterior approach (9), lateral approach $(10)$, caudal approach $(11,12)$, hand-assisted approach (13), robotic liver resection (14) or spacers-based approach (15), have been discussed. Intercostal ports and postural changes (16), including left lateral positioning and semi-prone positioning, have also been used to facilitate access to the target area, in combination with mobilization of the liver. However, these approaches remain imperfect; for example, the caudal approach, which does not require the dissociation of the perihepatic ligaments, is more suitable for major right hepatectomy. Improved, easier and more economically viable methods require development in order to improve the procedure of LRPH. After years of laparoscopic surgical investigation 
and using evidence-based research, our center has developed a systematic LRPH procedure, which is suitable for right partial hepatectomy, particularly for tumors located in the deep S VI, $\mathrm{S}$ VII or S VIII. This approach includes seven key points and three main instruments. All the surgical devices are affordable, commonplace and easy to obtain. Therefore, the present study analyzed our LRPH surgical outcomes using this approach to demonstrate and share its feasibility and superiority. A total 52 cases were included in this study. Excluding hepatocellular carcinoma, hemangioma and other tumors were also included, due to their indications for partial hepatectomy. The value, safety and quality of the surgical procedure were assessed.

\section{Materials and methods}

Clinical materials. Between June 2013 and April 2017, >260 LHs were performed at the Department of General Surgery, Qilu Hospital of Shandong University (Jinan, China). A total of 52 LRPHs and 29 open hepatectomies (OHs) were included in the present study, consisting of 55 females and 26 males. The mean age was $48.96 \pm 11.48$ years in the LRPH group and $54.62 \pm 8.76$ years in the $\mathrm{OH}$ group. A novel difficulty scoring system (17), which was created for discussion at the International Consensus Conference on Laparoscopic Liver Resection, was used for reference. Strict inclusion criteria were set up based on the histopathology of the lesion, size of the lesion, Child-Pugh grade (18), age and sex of the patient. The exclusion criteria included the following: Child-Pugh grade $\mathrm{C}$, ascites, cardiac failure, lung function insufficiency, renal dysfunction, history of prior hepatectomy and concomitant major operative procedures during hepatic resection (i.e., splenectomy, bowel resection, adrenal gland resection or esophageal devascularization).

Preoperative assessments included chest X-ray, abdominal B-ultrasonic examination, and computed tomography (CT) and magnetic resonance imaging (MRI) scans. Post-operative complications were graded according to the Clavien-Dindo classification (19).

The present study was approved by the Ethical Committee of 302 Military Hospital of China (Beijing, China) to conform with institutional deadlines. Due to the retrospective nature of the study, the requirement of written informed consent was waived.

LRH procedures. All procedures were performed under general anesthesia. Patients were placed in a supine position, with a surgeon on the right-hand side and a surgeon-assistant and camera-assistant on the left-hand side. The systematic '7+3' approach was used in all LRPHs, based on procedures described previously $(15,20-22)$, including low central venous pressure (CVP), intermittent clamping of the hepatic pedicle, pneumoperitoneum at $12 \mathrm{mmHg}$, parenchymal section with ultrasonic dissector and sterile glove pouch. The seven key points include the following: Special triangular positioning (15,23-27), lifting the right arm, rotating internally with an angle of $90^{\circ}$ elbow flexion fixed on the support and elevating the right side of the body at an angle of $15-30^{\circ}(\beta)$ inclined to left, which looks like a triangle (Fig. 1); improved trocar location (9), the observation port used in the present study was located to the right of the umbilicus, the main operating port was located in the right collarbone midline and the right axillary front-line at the same level as the umbilicus, and the assistant ports were located below the process and at the midpoint between the xiphoid process and the umbilicus; the 'grasping and pulling' method (Fig. 2) to establish the pneumoperitoneum (28), grasping the right abdominal wall of the umbilicus and pulling up, prior to using a Verres needle to establish pneumoperitoneum with a specific vertical angle, at which the right side of the body is elevated of $15-30^{\circ}$ and inclined to the left; the 'water sac' method (Fig. 3)to underlay the liver $(15,29)$, a sterile glove was placed into the abdominal cavity through a port and, following perfusion with saline, a suture was used to tie the orifice of the sterile glove to form a pouch, which was padded behind the right side of the liver to enhance surgical field exposure; modified hepatic portal occlusion (30,31), the main portion (Fig. 4) was created from the neck of an ultrasonic scalpel (YZB/USA 7411-2013, Ethicon Endo-Surgery, LLC, Guaynabo, Puerto Rico). Size 14\# or 16\# T tubes (201603G3, Zhanjiang Star Enterprise Co., Ltd., Zhanjiang, Guangdong, China) were used to form the buffer part of the device to reduce vessel and tissue damage, and block band was applied to encircle the hepatic portal (Fig. 5), then whole device punctures into the abdominal cavity partly through an additional small hole above the umbilicus; the 'hug' method to facilitate better exposure, a ribbon gauze was used to surround and move the liver, acting like a 'hug', which may improve exposure of the ligaments around the bare area and the second hepatic hilum (Fig. 6); and 'clamp crushing' method to dissect the hepatic parenchyma $(32,33)$, an ultrasonic scalpel was used to crush the soft liver parenchyma until only the Glisson sheath and vessels are remaining. They were subsequently clamped using a hemo-lock prior to dissection (Fig. 7). The three main instruments comprise laparoscopic forceps, a laparoscopic aspirator and an ultrasonic scalpel.

Statistical analysis. Data are presented as the mean \pm standard deviation, or as percentages. Continuous variables were compared using Student's t-test where appropriate. Categorical variables were analyzed using the $\chi^{2}$ test where appropriate. If the values were not normally distributed, a rank test was used. Survival, measured as the time and rate, was calculated using the Kaplan-Meier method and differences between the two groups were compared using the log-rank test. $\mathrm{P}<0.05$ was considered to indicate a statistically significant difference. All measurements and calculations were analyzed using SPSS software version 20 (IBM Corp., Armonk, NY, USA).

\section{Results}

Demographic profiles. A total of 52 LRPHs and 29 OHs were examined in the present study. The demographic profiles of patients are presented in Table I. The mean tumor size was $6.44 \mathrm{~cm}$ in the LRPH group and $6.47 \mathrm{~cm}$ in the $\mathrm{OH}$ group. No significant differences were observed between the two groups. All the cases were Child-Pugh Grade A and all the tumors were located in the deep S VI, S VII or S VIII.

Surgery-associated parameters. Surgical parameters and the postoperative recovery of patients are presented in Table II. Blood loss $(245.38 \pm 268.37 \mathrm{ml})$ in the LRPH group was not significantly more than that in the $\mathrm{OH}$ group $(230.93 \pm 257.62 \mathrm{ml}$; 
Table I. The demographic profiles of patients.

\begin{tabular}{|c|c|c|c|}
\hline Characteristic & LPRH $(n=52)$ & $\mathrm{OH}(\mathrm{n}=29)$ & P-value \\
\hline $\operatorname{Sex}(\%)$ & & & 0.114 \\
\hline Female, n & $35(67.3)$ & $20(69.0)$ & \\
\hline Male, $\mathrm{n}$ & $17(32.7)$ & $9(31.0)$ & \\
\hline Age, years & $48.96 \pm 11.48$ & $54.62 \pm 8.764$ & 0.064 \\
\hline Tumor size, $\mathrm{cm}$ & $6.44 \pm 3.11$ & $6.47 \pm 3.13$ & 0.094 \\
\hline Child-Pugh (A/B), n & $52 / 0$ & $29 / 0$ & \\
\hline Pathology (\%) & & & 0.037 \\
\hline $\mathrm{HCC}, \mathrm{n}$ & $23(44.2)$ & $22(75.9)$ & \\
\hline Hemangioma, $\mathrm{n}$ & $23(44.2)$ & $6(20.7)$ & \\
\hline Metastasis, $\mathrm{n}$ & $2(3.8)$ & $1(3.4)$ & \\
\hline Other, $\mathrm{n}$ & $4(7.7)$ & $0(0)$ & \\
\hline Location (\%) & & & 0.870 \\
\hline $\mathrm{VI}, \mathrm{n}$ & $21(40.4)$ & $5(17.2)$ & \\
\hline VII, n & $12(23.1)$ & $7(24.1)$ & \\
\hline VIII, n & $2(3.8)$ & $3(10.3)$ & \\
\hline VI, VII, n & $14(26.9)$ & $7(24.1)$ & \\
\hline VII, VIII, n & $2(3.8)$ & $5(17.2)$ & \\
\hline VI, VII, VIII, n & $1(1.9)$ & $2(7.0)$ & \\
\hline
\end{tabular}

Data are presented as the mean \pm standard deviation, numbers or percentages. LRPH, laparoscopic right partial hepatectomy; $\mathrm{OH}$, open hepatectomy; HCC, hepatocellular carcinoma.

$\mathrm{P}=0.936)$. The durations of surgery, liver parenchyma transection and portal triad clamping time were also not significantly more than those in the $\mathrm{OH}$ group $(145.52 \pm 48.29$ vs. $129.83 \pm 35.04 \mathrm{~min} ; \mathrm{P}=0.149$ for surgery; $28.52 \pm 10.16$ vs. $23.97 \pm 10.44 \mathrm{~min} ; \mathrm{P}=0.059$ for liver parenchyma transection; and $20.62 \pm 9.61$ vs. $17.31 \pm 10.12 \mathrm{~min}, \mathrm{P}=0.149$ for portal triad clamping). However, the number of postoperative hospital days in the LRPH group was lower than that in the $\mathrm{OH}$ group (10.67 in the LRPH group vs. 12.07 in the $\mathrm{OH}$ group; $\mathrm{P}=0.025)$. In the present study, 18 cases required repeated clamping [11 (21.2\%) in the LRPH group vs. 7 (24.1\%) in the $\mathrm{OH}$ group]. There were no intraoperative mortalities or morbidities requiring repeated surgery. None of the patients succumbed within 90 days after the operation. Postoperative complications occurred in 10 patients, including 5 cases of bile leakage, 2 cases of liver dysfunction, 1 case of hemorrhage, 1 case of intestinal obstruction and 1 case of biloma. However, according to the Clavien-Dindo classification, the severity of complications was not significantly different between the LRPH and $\mathrm{OH}$ groups (11.5 vs. 13.8\%; $\mathrm{P}=0.679)$. Only 3 (5.8\%) conversions to open surgery occurred, proving that the success rate of this approach was high.

Long-term outcomes. The follow-up period was $\sim 4$ years. A total of $23(44.2 \%)$ patients in the LRPH group were diagnosed with hepatocellular carcinoma (HCC), while $22(75.9 \%)$ patients in the $\mathrm{OH}$ group were diagnosed with HCC. The pathology data differed significantly between the two groups $(\mathrm{P}=0.037)$. In order to obtain improved surgical results, only patients with HCC were selected for the study of long-term outcomes. The 4-year overall survival and relapse-free survival rates were 78.3 and $47.8 \%$, and 72.7 and $40.9 \%$ for the LRPH and the $\mathrm{OH}$ groups, respectively (Fig. 8), and there were no significant difference between the two groups (OS, $\mathrm{P}=0.670$; RFS, $\mathrm{P}=0.659$ ).

\section{Discussion}

Since the first International Consensus Conference on Laparoscopic Liver Resection (34), LH has been rapidly adopted all over the world. However, for LRPH located in the deep S VI, S VII or S VIII, there are numerous limitations, not only due to the restricted manipulation, but also as a result of restricted exposure. The aim of the present study was to form a novel systematic method to aid in performing LRPHs more efficiently. A total of $52 \mathrm{LRPH}$ and $29 \mathrm{OH}$ cases were selected for certain comparative analyses. Although the operations in the LRPH group were technically demanding more frequently than in the $\mathrm{OH}$ group, the duration of surgery, clamping time, blood loss, intraoperative transfusion rate, complication rate, severity, OS and RFS were not significantly different between the two groups. In the LRPH group, the duration of postoperative hospital stay was shorter than that in the $\mathrm{OH}$ group (10.67 in the LRPH group vs. 12.07 in the $\mathrm{OH}$ group; $\mathrm{P}=0.025)$. The open conversion rate in the present study was low (5.8\%). No mortalities occurred in 90 days. All the results of the present study reflected the feasibility and safety of this approach.

LRPH is considered to be a challenging procedure to perform, even for experienced surgeons (35). The main difficulty is the poor operative field, associated with difficulties in achieving adequate tumor margins and in controlling bleeding. Certain surgeons have reported that an anterior or caudal approach without mobilization is a preferable method for major right hepatectomy $(9,12)$. However, we hypothesized that complete mobilization of the right side of the liver remains an important assurance for right partial hepatectomy, particularly in posterior segments. Our previous study also discussed this hypothesis (15). Therefore, certain modifications were made in the present study in order to develop a systematic surgical approach to overcome these limitations. It is not a novel technique, but a combination and improvement on several factors demonstrated to be associated with improved liver exposure and decreased blood loss during hepatectomy.

The special triangular position. Surgical view and organ manipulation is restricted in the regular caudal approach to LH. In order to solve this problem, postural changes, which have been reported in previous studies, may be applied, including the left lateral position for posterior sectionectomy (23) and the semi-prone position for tumors located in the posterosuperior segments $(24,25)$. The reverse Trendelenburg position (26) and rotation of the body to as close to the left lateral decubitus position as possible $(15,27)$ aid in decreasing venous pressure and improving exposure by gravitationally shifting visceral structures away from the hepatic hilum. The weight of the liver facilitates the mobilization of the liver itself, ultimately creating a space above the liver. Furthermore, since the posterior section is positioned higher than the inferior vena cava, 
Table II. Operative parameters and postoperative recovery of patients.

\begin{tabular}{|c|c|c|c|}
\hline Variable & LRPH $(n=52)$ & $\mathrm{OH}(\mathrm{n}=29)$ & P-value \\
\hline Operation time, $\min$ & $145.52 \pm 48.29$ & $129.83 \pm 35.04$ & 0.130 \\
\hline Time of clamping porta hepatis, min & $20.62 \pm 9.61$ & $17.31 \pm 10.12$ & 0.149 \\
\hline Repeated clamping, n (\%) & $11(21.2)$ & $7(24.1)$ & \\
\hline No clamping, n (\%) & $7(13.5)$ & $5(17.2)$ & \\
\hline Parenchyma dissection time, min & $28.52 \pm 10.16$ & $23.97 \pm 10.44$ & 0.059 \\
\hline Blood loss, ml & $245.38 \pm 268.37$ & $230.93 \pm 257.62$ & $0.936^{\mathrm{a}}$ \\
\hline Conversion, n (\%) & $3(5.8)$ & - & \\
\hline Transfusion, n (\%) & $6(11.5)$ & $3(10.3)$ & 0.870 \\
\hline Postoperative hospital day, days & $10.67 \pm 3.32$ & $12.07 \pm 3.22$ & $0.025^{\mathrm{a}}$ \\
\hline Morbidity, n (\%) & $5(10)$ & $4(13.8)$ & 0.566 \\
\hline day mortality rate, n (\%) & $0(0)$ & $0(0)$ & \\
\hline Number of complications, n (\%) & $6(11.5)$ & $4(13.8)$ & 0.679 \\
\hline \multicolumn{4}{|l|}{ Clavien-Dindo scoring, $\mathrm{n}$} \\
\hline I & 0 & & \\
\hline II & $\begin{array}{l}4 \text { (2 Bile leakage, } 1 \text { liver dysfunction, } \\
1 \text { hemorrhage) }\end{array}$ & $\begin{array}{l}2 \text { (Bile leakage, } \\
\text { intestinal obstruction) }\end{array}$ & \\
\hline III & 2 (Bile leakage, biloma) & 1 (Bile leakage) & \\
\hline IV & 0 & 1 (Liver dysfunction) & \\
\hline $\mathrm{V}$ & 0 & & \\
\hline
\end{tabular}

${ }^{a}$ Rank test. LRPH, laparoscopic right partial hepatectomy; $\mathrm{OH}$, open hepatectomy. The values are expressed as the mean \pm standard deviation, numbers or percentages.

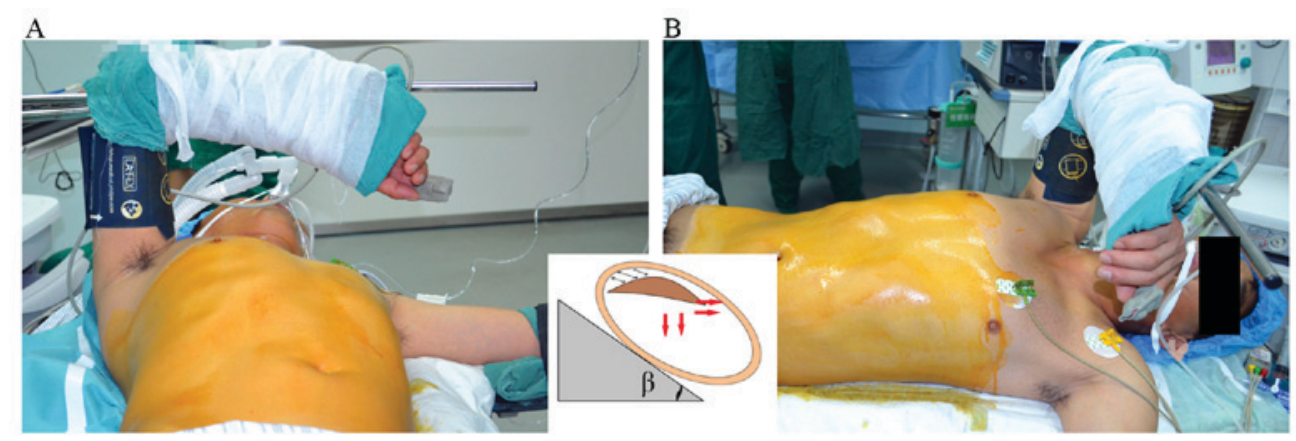

Figure 1. The triangular position. The right arm is listed and rotated internally with an angle of $90^{\circ}$ elbow flexion fixed on the support and the right side of the body is elevated with an angle of $15-30^{\circ}(\beta)$ inclined to the left. (A) Norma inferior; (B) norma lateralis.

less intraoperative bleeding occurs and the irrigation fluids and blood flow down to the lower left side, facilitating a good view of the operative field. Based on this, the patient position used in the present study was adjusted (Fig. 1). A preclinical study has demonstrated that this position may aid in decreasing bleeding and improving liver exposure (15). In the present study, all the LRPHs were performed in this position and decreased levels of bleeding were observed.

The improved trocar sites-enlarged reverse ' $L$ ' set. Trocar sites determine the success of LH. In general, five trocars were used: Three of 5-mm diameter, one of 10-mm diameter and one of 12-mm diameter. Derived from traditional trocar sites (9), our sites were distributed in a sites-enlarged reverse ' $\mathrm{L}$ ' form. When conversion was required, the ' $\mathrm{L}$ ' incision form was reversed. The trocar areas were extended compared with traditional sites, which may aid in forming a larger operative space, and gaining a clearer overhead view of the operative field and the hepatic portal area, which is an important anatomical structure in hepatectomy, and in providing a clear view of the dorsal side of the liver from the inferior side (Fig. 2). The main operating port may also be used as an observation port to obtain an improved view of the dorsal side of the liver.

Establishing pneumoperitoneum using the 'grasping and pulling' method. There are numerous ways to establish pneumoperitoneum, including the Verres and Hasson technique (28), with different advantages and disadvantages. It was reported that the incidence of intra-abdominal injury was $0.13 \%$ in patients who had undergone prior abdominal surgery, 

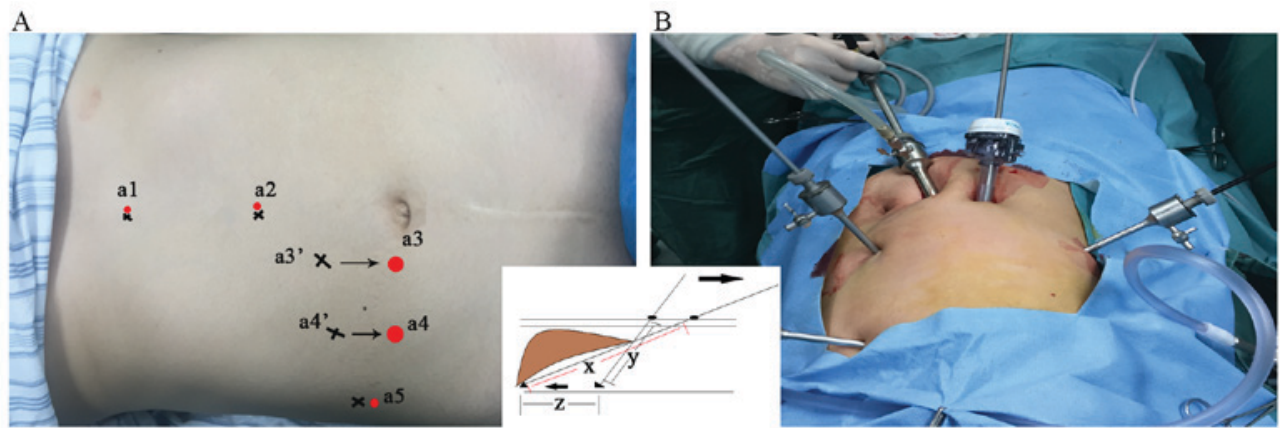

Figure 2. Trocar set. Enlarged reverse 'L' shape of the trocar set; three of 5-mm diameter (a1, a2 and a5), one of 10-mm diameter (a4) and one of 12-mm diameter (a5). The changes (a3'-a3 and a4'-a4) enlarge the operative field, with an increase in the visual depth ( $\mathrm{z}$ ) and an increase in the moving range ( $\mathrm{x}>\mathrm{y})$. (A) Before placement; (B) after placement.
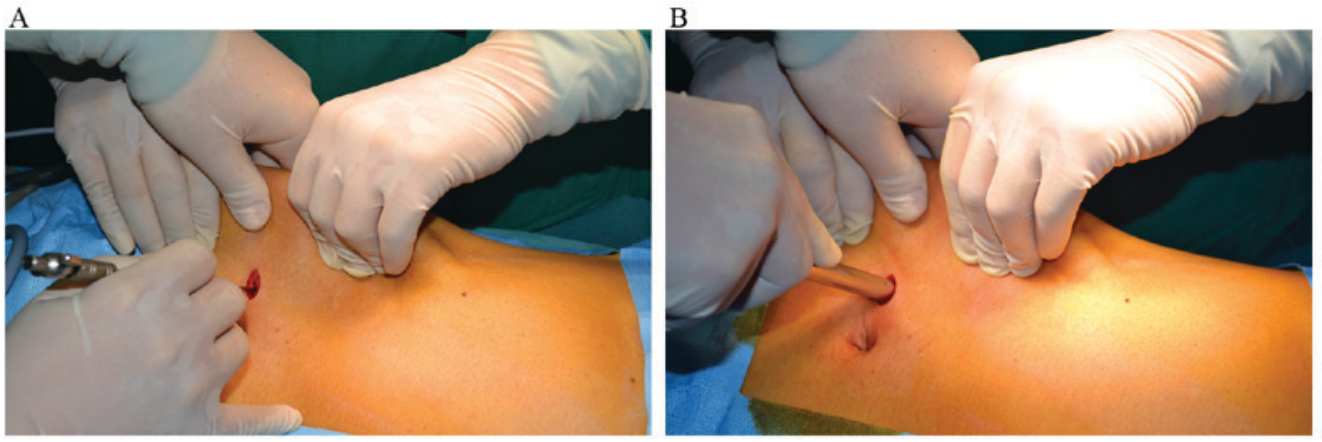

Figure 3. The 'grasping and pulling' method is used to establish pneumoperitoneum. The right abdominal wall of the umbilicus was grabbed and pulled up, prior to a Verres needle being used to establish pneumoperitoneum perpendicular to the abdominal wall. (A) The puncturing of needle; (B) the placement of trocar.
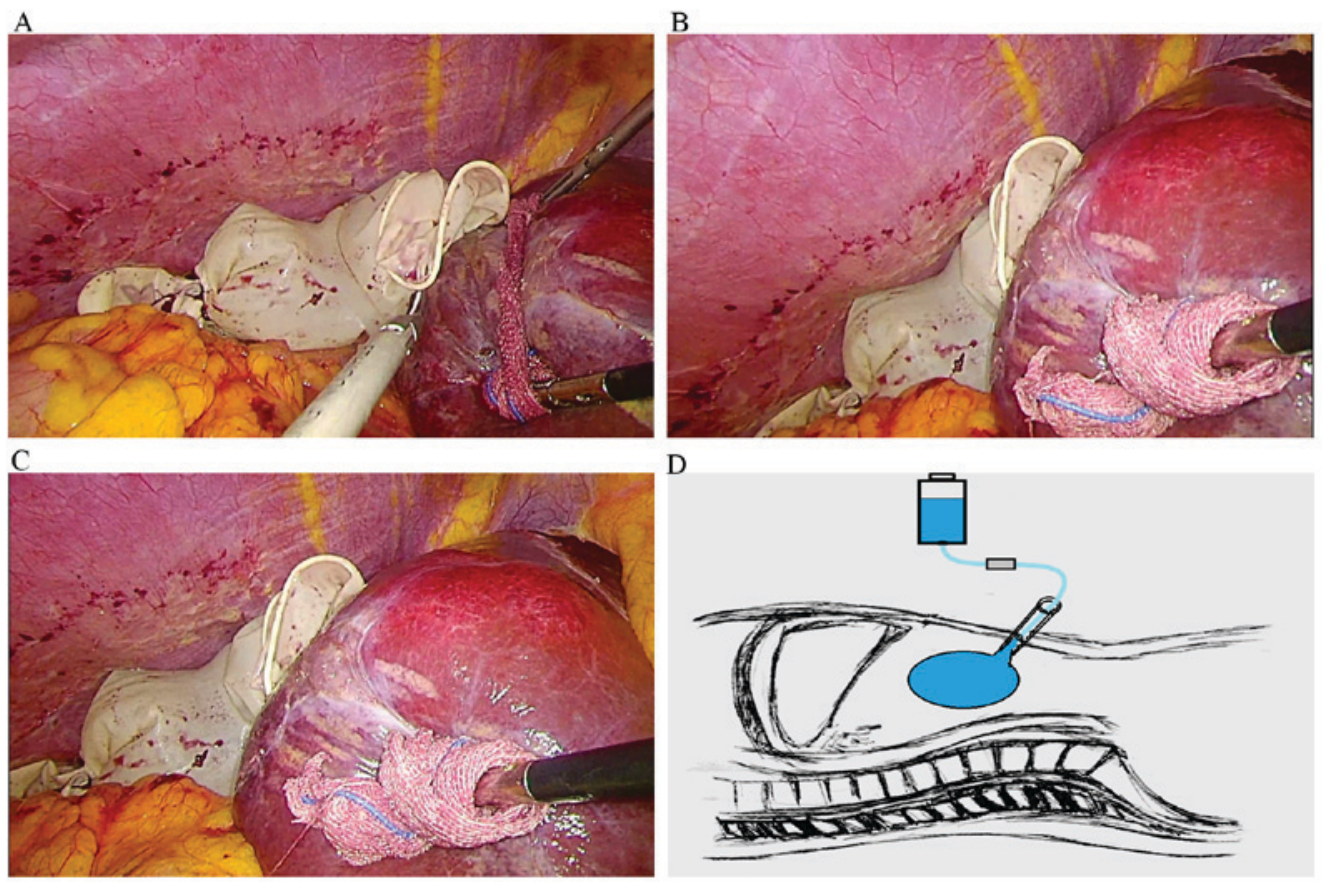

Figure 4. The 'water sac' method is used to underlay the liver. (A) A sterile glove was placed into the abdominal cavity through a port and it was perfused with saline. (B) The orifice was then tied to form a pouch, which was subsequently (C) padded behind the right side of the liver to enhance the surgical field exposure. (D) The saline injection process.

and in the subgroup of patients who had undergone previous surgery the rate of intra-abdominal injury was $0.78 \%$; while no associated injuries occurred in the present study, which demonstrated the safety of the method. The traditional Verres needle technique requires the use of towel forceps, which may lead to other subsidiary-injuries. In order to pursue a less 
A

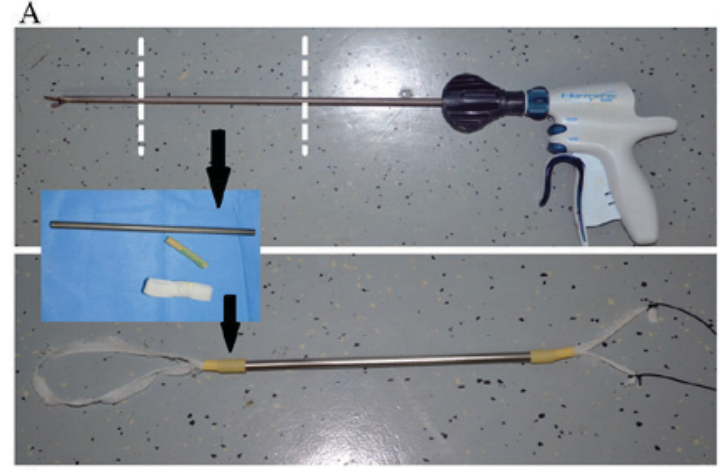

$\mathrm{C}$

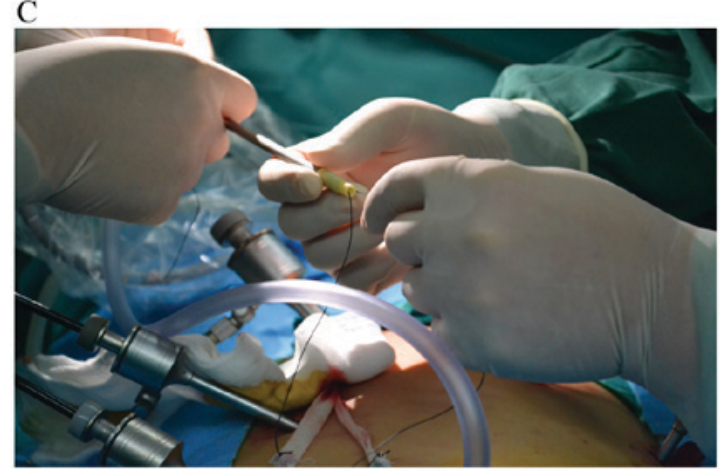

B

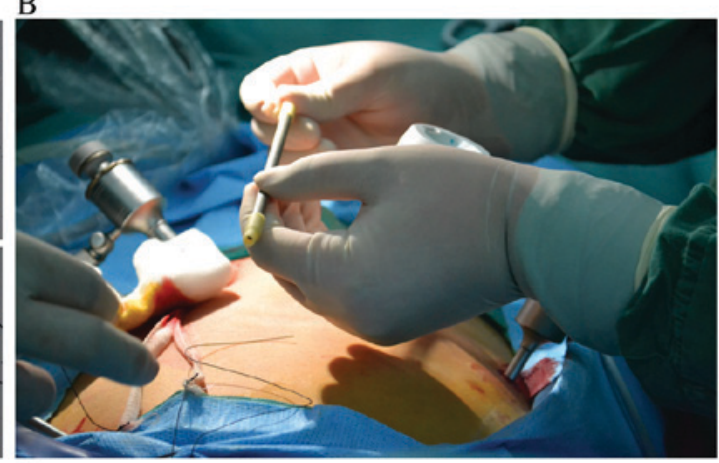

D

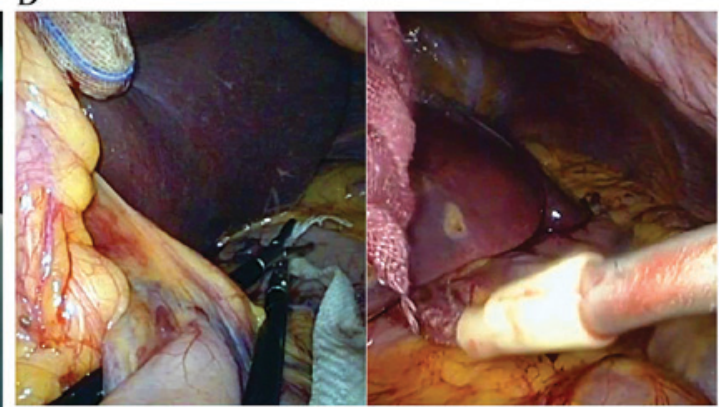

Intra-abdominal visual field

Figure 5. The 'controllable' method is used to control hepatic portal occlusion. The instrument used was constructed primarily from an ultrasonic scalpel. The components were shown in part A. (B) A size 14\# or $16 \# \mathrm{~T}$ tube was placed on both sides of the metal tube and used to form the buffer part to reduce vessel and tissue damage. (C) A \#7 silk suture, attached a block band, was crossed through the tube. (D) The tube penetrated the abdominal wall and the block band was used to encircle the porta hepatis.
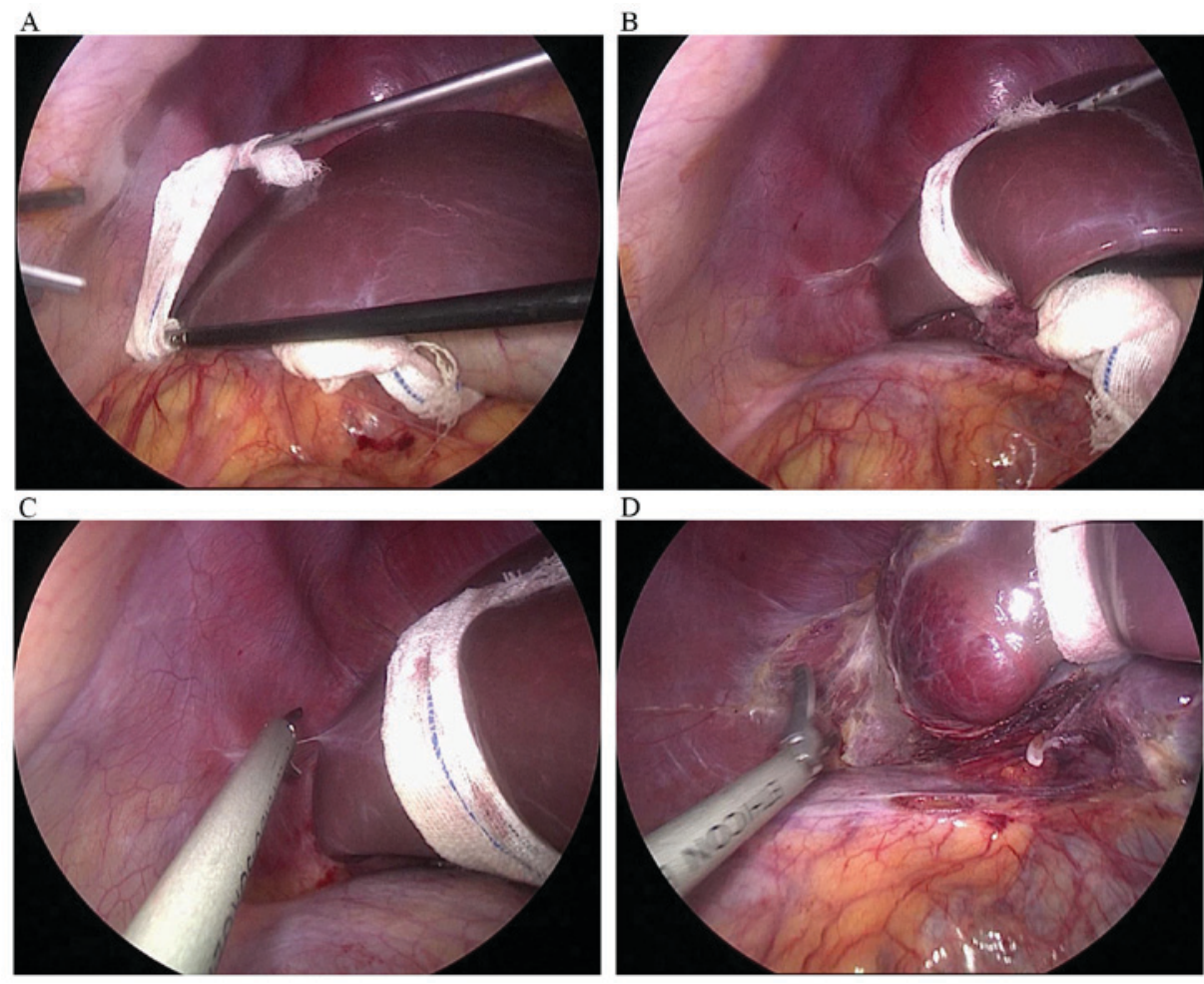

Figure 6. The 'hug' method is used to facilitate improved exposure. A ribbon gauze was utilized to surround and move the liver. (A) Place-(B) hug-(C) dissect(D) haul. Repeat.

invasive technique, a 'grasping and pulling' method (Fig. 3), which no longer requires towel forceps, was developed. The important points are grasping, pulling and the special vertical angle. It is not recommended to use the left side as the puncture point, in order to avoid the possibility of spleen injury in cases of splenomegaly. 
A

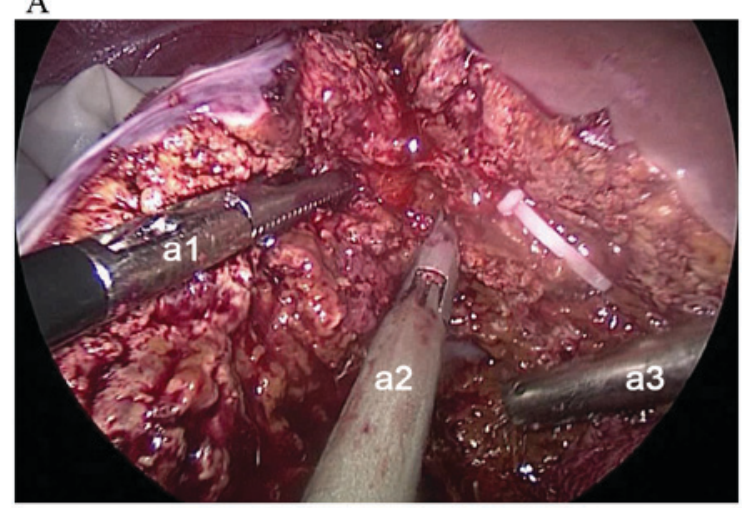

B

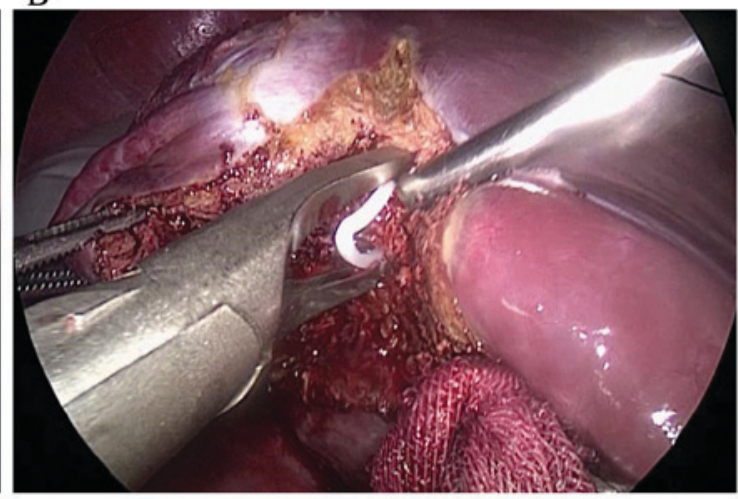

Figure 7. The 'clamp crushing' method is used to dissect the hepatic parenchyma. (A) An ultrasonic scalpel was used to crush the soft liver parenchyma until only the Glisson sheath and vessels are remaining. (B) They were subsequently clamped using a hemo-lock prior to dissection. a1, forceps; a2, ultrasonic scalpel; a3, aspirator.

A

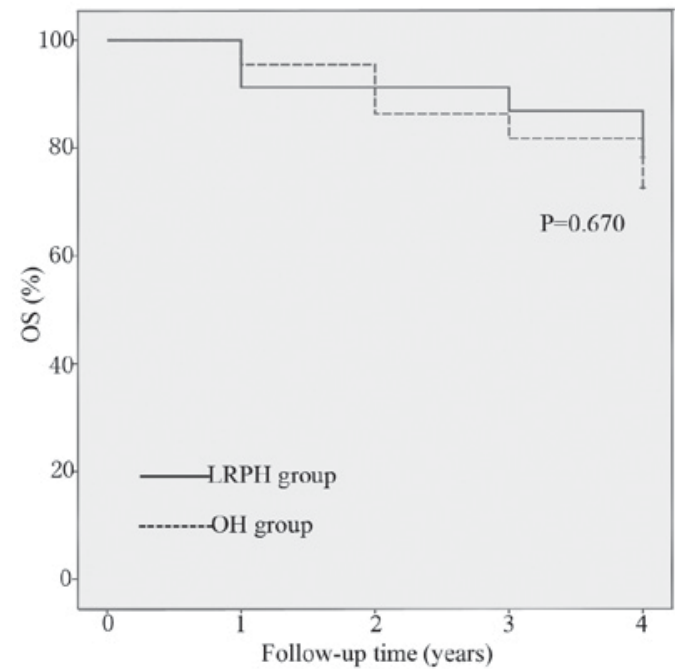

B

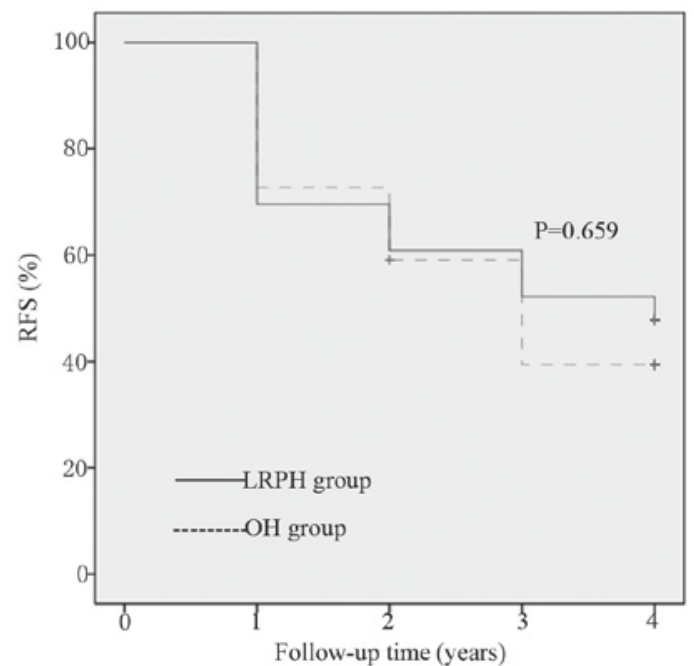

Figure 8. Comparison of the (A) OS and (B) RFS rates of patients with hepatocellular carcinoma between the LRPH and the OH groups. No significant differences were observed between the two groups (OS, $\mathrm{P}=0.670$; RFS, $\mathrm{P}=0.659$ ). OS, overall survival; RFS, recurrence-free survival; LRPH, laparoscopic right partial hepatectomy; $\mathrm{OH}$, open hepatectomy.

The use of the 'water sac' method to underlay the liver. The posterior side of the liver is difficult to expose when performing a right hepatectomy (29). This is a key reason why LRPHs are challenging to perform. For improved exposure, the 'water sac' method (Fig. 4) was created at the Qilu Hospital of Shandong University. A study has been performed to prove the superiority of this technique (15), the results of which have suggested that this method may enhance exposure in the surgical field, and result in decreased blood loss and procedure time. In addition, the saline may be used to irrigate the surgical field by puncturing the glove pouch at the end of surgery. In our previous study, the durations of liver parenchyma transection and portal triad clamping were significantly shorter compared with those in a control laparoscopic group without a sterile glove pouch $(30.29 \pm 5.55$ vs. $39.00 \pm 3.68 \mathrm{~min}, \mathrm{P}<0.001$ for liver parenchyma transection; and $23.00 \pm 5.60$ vs. $31.60 \pm 5.03 \mathrm{~min}$, $\mathrm{P}<0.001$ for portal triad clamping) (15). As the method was improved, improved results were obtained: In the present study, the time of liver parenchyma transection and portal triad clamping was $28.52 \pm 10.16 \mathrm{~min}$ and $20.62 \pm 9.61 \mathrm{~min}$, respectively.

The modified 'controllable' hepatic portal occlusion method. With the exception of the half hepatic portal clamping (30) performed in the right anatomical hemihepatectomy, the Pringle maneuver (31) was selected to strive for the shortest surgery duration. The mean duration of hepatic portal clamping was $20.62 \mathrm{~min}$, with 11 (21.2\%) patients requiring the application of more than one period of controlled occlusion. This high rate demonstrated that it was necessary to identify a way to control the occlusion in order to achieve repeated clamping during the surgery. Subsequently, a 'controllable' method was developed. The clamping device used in the present study was also self-made (Fig. 5). According to the requirements of the surgery, the band-blockade may be controlled extra-corporeally to obtain controllable clamping. Using this method, a similar clamping time and repeated clamping rate was obtained between the LRPH and $\mathrm{OH}$ groups $(\mathrm{P}=0.149)$. 
The 'hug' method was used to ensure improved liver exposure. As mentioned earlier, the issue of liver exposure has restricted the development of LRPH. To manipulate the liver during $\mathrm{OH}$, the surgeon opens the subphrenic cage with a large subcostal incision, lifts up the costal arch and physically picks up the liver with the left hand following dissection of the retro-peritoneal attachments. In LH, however, there are no instruments that are as precise as a surgeon's hand, and it is not possible to access the anterior space of the liver without an abdominal wall incision. The ligaments around the anterior area are difficult to see. The authors' 'hug' method may improve exposure of the ligaments around the bare area and the second hepatic hilum (Fig. 6). The feasibility and superiority of this method were greatly supported by the similar blood loss, and the durations of surgery, liver parenchyma transection and portal triad clamping time.

The 'clamp crushing' method was used to dissect the hepatic parenchyma. The 'clamp crushing' technique was introduced in the 1980s (32), following which it became the gold standard for liver parenchymal transection in $\mathrm{OH}$ due to its effectiveness in controlling blood loss and reducing the duration of surgery, and its low cost (33). In the LRPHs performed in the present study, a 'clamp crushing' technique was used with an ultrasonic scalpel (Fig. 7). Through careful dissection, the bleeding and duration of surgery were well controlled. In the present study, the bleeding and duration of surgery were not significantly different between the LRPH and OH groups.

Despite the difficult procedures used in the LRPH group, the complication rates and Clavien-Dindo grades were not significantly different between the two groups. As aforementioned, the pathology data differed significantly between the two groups $(\mathrm{P}=0.037)$, and so in order to obtain improved surgical results, only patients with HCC were selected for the study of long-term outcomes. As mentioned earlier, the OS and RFS rates were 78.3 and $47.8 \%$, and 72.7 and $40.9 \%$ in the LRPH and OH groups, respectively, and no significant differences were observed (Fig. 8). Therefore, the approach utilized in the present study is a feasible and safe method and the difficulty of the surgery should not be regarded as a contraindication. However, the surgical team must accumulate further experience with this technique. The learning curves presented in previous studies have recommended that at $\geq 30-60$ surgeries be performed to ensure the stability of LH $(36,37)$.

Newer types of surgical instruments are available, including bipolar coagulation, LigaSure, bipolar dissecting forceps and ENDO CUT. Due to their convenience, these instruments have been generally praised and used by surgeons. However, this may add to the associated cost of surgery, which may influence the popularization and promotion of LH in primary hospitals. The present study attempted to simplify the surgical procedure and instruments in order to reduce surgical expense as much as possible. Three main pieces of apparatus were used in the hepatic parenchyma transection, including one ultrasonic scalpel and two normal laparoscopic instruments, forceps and an aspirator [Fig. 8A (a1/a2/a3)]. The associated results proved that this was feasible. Almost all the hepatic parenchyma transections were accomplished very well using just these three main instruments, enabling positive surgical outcomes to be achieved. The duration of liver parenchyma transection in the LRPH group was not significantly longer than that in the $\mathrm{OH}$ group $(\mathrm{P}=0.059)$.

Furthermore, it is possible to create the majority of devices used in this procedure on-site and/or to use them repeatedly, which would reduce the cost of the LH.

The present study demonstrated the satisfactory surgical outcomes and economic benefits of the systematic ' $7+3$ ' surgical procedure for LRPH. The use of seven key points and three main instruments could be mastered easily by the majority of surgeons and the simplicity, convenience and low cost of this technique could be accepted by the majority of primary hospitals. This would improve the popularity of LH and change the current situation in which LH may only be performed in certain tertiary hospitals. Further studies in larger cohorts and other centers are required to confirm the feasibility of this technique and its superiority to current methods.

\section{Acknowledgements}

Not applicable.

\section{Funding}

The present study was supported by the Fundamental Research Funds of Shan Dong University (Qilu Hospital Research Project; grant no. 2014QLKY18) and the Natural Science Foundation of China (grant no. 81571367).

\section{Availability of data and materials}

The datasets used during the current study are available from the corresponding author on reasonable request.

\section{Authors' contributions}

JL and HR analyzed and interpreted the data regarding LRPH and $\mathrm{OH}$. GD collected the data regarding LRPH and OH. BJ provided substantial contributions to conception and design of the study, and revised it critically for important intellectual content. All authors read and approved the final manuscript.

\section{Ethics statement and consent to participate}

The study was approved and consented by the Ethical Committee of 302 Military Hospital of China (Beijing, China).

\section{Consent for publication}

Consent was obtained for the publication of the patient's data and images.

\section{Competing interests}

The authors declare that they have no competing interests.

\section{References}

1. Nguyen KT, Gamblin TC and Geller DA: World review of laparoscopic liver resection-2,804 patients. Ann Surg 250: 831-841, 2009. 
2. Cherqui D, Laurent A, Mocellin N, Tayar C, Luciani A, Van Nhieu JT, Decaens T, Hurtova M, Memeo R, Mallat A and Duvoux C: Liver resection for transplantable hepatocellular carcinoma: Long-term survival and role of secondary liver transplantation. Ann Surg 250: 738-746, 2009.

3. Sasaki A, Nitta H, Otsuka K, Takahara T, Nishizuka S and Wakabayashi G: Ten-year experience of totally laparoscopic liver resection in a single institution. Br J Surg 96: 274-279, 2009.

4. Dagher I, O'Rourke N, Geller DA, Cherqui D, Belli G, Gamblin TC, Lainas P, Laurent A, Nguyen KT, Marvin MR, et al: Laparoscopic major hepatectomy: An evolution in standard of care. Ann Surg 250: 856-860, 2009.

5. Belli G, Gayet B, Han HS, Wakabayashi G, Kim KH, Cannon R, Kaneko H, Gamblin T, Koffron A, Dagher I, et al: Laparoscopic left hemihepatectomy a consideration for acceptance as standard of care. Surg Endosc 27: 2721-2726, 2013.

6. Ciria R, Cherqui D, Geller DA, Briceno J and Wakabayashi G: Comparative short-term benefits of laparoscopic liver resection: 9000 cases and climbing. Ann Surg 263: 761-777, 2016.

7. Cai X, Li Z, Zhang Y, Yu H, Liang X, Jin R and Luo F. Laparoscopic liver resection and the learning curve: A 14-year, single-center experience. Surg Endosc 28: 1334-1341, 2014.

8. Cheng KC, Yeung YP, Ho KM and Chan FK: Laparoscopic right posterior sectionectomy for malignant lesions: An anatomic approach. J Laparoendosc Adv Surg Tech A 25: 646-650, 2015.

9. Takahashi M, Wakabayashi G, Nitta H, Takeda D, Hasegawa Y, Takahara $\mathrm{T}$ and Ito N: Pure laparoscopic right hepatectomy by anterior approach with hanging maneuver for large intrahepatic cholangiocarcinoma. Surg Endosc 27: 4732-4733, 2013.

10. Kim JH, Ryu DH, Jang LC and Choi JW: Lateral approach liver hanging maneuver in laparoscopic anatomical liver resections. Surg Endosc 30: 3611-3617, 2016.

11. Tomishige H, Morise Z, Kawabe N, Nagata H, Ohshima H, Kawase J, Arakawa S, Yoshida R and Isetani M: Caudal approach to pure laparoscopic posterior sectionectomy under the laparoscopy-specific view. World J Gastrointest Surg 5: 173-177, 2013.

12. Soubrane O, Schwarz L, Cauchy F, Perotto LO, Brustia R, Bernard D and Scatton O: A conceptual technique for laparoscopic right hepatectomy based on facts and oncologic principles: The caudal approach. Ann Surg 261: 1226-1231, 2015.

13. Makdissi FF, JeismannVB, Kruger JAP, Coelho FF, Ribeiro-Junior U, Cecconello I and Herman P: Hand-assisted approach as a model to teach complex laparoscopic hepatectomies: Preliminary results. Surg Laparosc Endosc Percutan Tech 27: 285-289, 2017

14. Choi GH, Chong JU, Han DH, Choi JS and Lee WJ: Robotic hepatectomy: The Korean experience and perspective. Hepatobiliary Surg Nutr 6: 230-238, 2017.

15. Bin J, Binghai $\mathrm{Z}$ and Sanyuan H: liver exposure using sterile glove pouch during laparoscopic right liver surgery in hepatocellular carcinoma patients. World J Surg 40: 946-950, 2016.

16. Lee W, Han HS, Yoon YS, Cho JY, Choi Y, Shin HK, Jang JY, Choi H, Jang JS and Kwon SU: Comparison of laparoscopic liver resection for hepatocellular carcinoma located in the posterosuperior segments or anterolateral segments: A case-matched analysis. Surgery 160: 1219-1226, 2016.

17. Ban D, Tanabe M, Ito H, Otsuka Y, Nitta H, Abe Y, Hasegawa Y, Katagiri T, Takagi $\mathrm{C}$, Itano $\mathrm{O}$, et al: A novel difficulty scoring system for laparoscopic liver resection. J Hepatobiliary Pancreat Sci 21: 745-753, 2014

18. Zheng J, Xing RC, Zheng WH, Liu W, Yao RC, Li XS, Du JP and Li L: A comparative study on postoperative mortality prediction of SFLI scoring system and Child-Pugh classification in patients with hepatocellular carcinoma. J BUON 22: 709-713, 2017.

19. Dindo D, Demartines N and Clavien PA: Classification of surgical complications: A new proposal with evaluation in a cohort of 6336 patients and results of a survey. Ann Surg 240 205-213, 2004

20. Ukere A, Meisner S, Greiwe G, Opitz B, Benten D, Nashan B, Fischer L, Trepte CJC, Reuter DA, Haas SA and Behem CR: The influence of PEEP and positioning on central venous pressure and venous hepatic hemodynamics in patients undergoing liver resection. J Clin Monit Comput 31: 1221-1228, 2017.

21. Rotellar F, Pardo F, Bueno A, Martí-Cruchaga P and Zozaya G: Extracorporeal tourniquet method for intermittent hepatic pedicle clamping during laparoscopic liver surgery: An easy, cheap, and effective technique. Langenbecks Arch Surg 397 481-485, 2012
22. Zhang X, Wei J, Song X, Zhang Y, Qian W, Sheng L, Shen Z, Yang L, Dong R and Gu W: Comparison of the impact of prolonged low-pressure and standard-pressure pneumoperitoneum on myocardial injury after robot-assisted surgery in the Trendelenburg position: Study protocol for a randomized controlled trial. Trials 17: 488, 2016.

23. Cho JY, Han HS, Yoon YS and Shin SH: Feasibility of laparoscopic liver resection for tumors located in the posterosuperior segments of the liver, with a special reference to overcoming current limitations on tumor location. Surgery 144: 32-38, 2008.

24. Ikeda T, Mano Y, Morita K, Hashimoto N, Kayashima H, Masuda A, Ikegami T, Yoshizumi T, Shirabe K and Maehara Y: Pure laparoscopic hepatectomy in semiprone position for right hepatic major resection. J Hepatobiliary Pancreat Sci 20: 145-150, 2013.

25. D'Hondt M, Yoshihara E, Vansteenkiste F, Steelant PJ, Van Ooteghem B, Pottel H, Devriendt D and Van Rooy F: Laparoscopic parenchymal preserving hepatic resections in semiprone position for tumors located in the posterosuperior segments. Langenbecks Arch Surg 401: 255-262, 2016.

26. Yoneda G, Katagiri S and Yamamoto M: Reverse Trendelenburg position is a safer technique for lowering central venous pressure without decreasing blood pressure than clamping of the inferior vena cava below the liver. J Hepatobiliary Pancreat Sci 22: 463-466, 2015.

27. Lee TE, Yoo C, Lin SC and Kim YY: Effect of different head positions in lateral decubitus posture on intraocular pressure in treated patients with open-angle glaucoma. Am J Ophthalmol 160: 929-936.e4, 2015.

28. Dunne N, Booth MI and Dehn TC: Establishing pneumoperitoneum: Verres or Hasson? The debate continues. Ann R Coll Surg Engl 93: 22-24, 2011.

29. Casaccia M, Andorno E, Di Domenico S, Santori G, Fazio F, Gelli $\mathrm{M}$ and Valente U: Laparoscopic right posterior sectionectomy for hepatocellular carcinoma using a modified liver-hanging maneuver. J Laparoendosc Adv Surg Tech A 22: 488-491, 2012.

30. Wijesurendere CN, Silva FH, Pathirana AA and Wijeratne T: Pringle manoeuvre versus selective hepatic vascular exclusion: Outcome of a case series in a tertiary referral centre. Ceylon Med J 59: 139-140, 2014.

31. Sanjay P, Ong I, Bartlett A, Powell JJ and Wigmore SJ: Meta-analysis of intermittent Pringle manoeuvre versus no Pringle manoeuvre in elective liver surgery. ANZ J Surg 83: 719-723, 2013.

32. Lin TY: Results in 107 hepatic lobectomies with a preliminary report on the use of a clamp to reduce blood loss. Ann Surg 177: 413-421, 1973

33. Chen JM, Geng W, Liu FB, Zhao HC, Xie SX, Hou H, Zhao YJ, Wang GB and Geng XP: BiClamp ${ }^{\circledR}$ forcep liver transection versus clamp crushing technique for liver resection: Study protocol for a randomized controlled trial. Trials 16: 201, 2015.

34. Buell JF, Cherqui D, Geller DA, O'Rourke N, Iannitti D, Dagher I, Koffron AJ, Thomas M, Gayet B, Han HS, et al: The international position on laparoscopic liver surgery: The Louisville statement, 2008. Ann Surg 250: 825-830, 2009.

35. Robinson SM, Hui KY, Amer A, Manas DM and White SA: Laparoscopic liver resection: Is there a learning curve? Dig Surg 29: 62-69, 2012.

36. Tomassini F1, Scuderi V, Colman R, Vivarelli M, Montalti R and Troisi RI: The single surgeon learning curve of laparoscopic liver resection: A continuous evolving process through stepwise difficulties. Medicine (Baltimore) 95: e5138, 2016.

37. Lin CW, Tsai TJ, Cheng TY, Wei HK, Hung CF, Chen YY and Chen CM: The learning curve of laparoscopic liver resection after the Louisville statement 2008: Will it be more effective and smooth? Surg Endosc 30: 2895-2903, 2016.

This work is licensed under a Creative Commons Attribution-NonCommercial-NoDerivatives 4.0 International (CC BY-NC-ND 4.0) License. 